\title{
SUCROSE IS A GOOD ALTERNATIVE SUSTAIN TO PROTECT PROTEIN STRUCTURE FOR ELECTRON CRYSTALLOGRAPHY
}

\author{
William H. Massover
}

Department of Cell Biology \& Molecular Medicine, UMDNJ - New Jersey Medical School, Newark, N.J. 07101-1709, USA

The ability of certain sugars to protect protein structure during drying or freezing is very useful to various organisms and industries [e.g., 1], as well as to electron crystallographers. Protein crystals commonly are prepared by drying within a solution of the monosaccharide, glucose [e.g., 2], the disaccharide, trehalose [e.g., 3], or the modified saccharide, tannate [e.g., 4]; these sugar sustains protect native protein structure from damage and denaturation during water removal. Using cryoprotection, transmission electron diffraction (TED) and microscopy (TEM) of several crystalline polypeptides dried within a sugar matrix have produced structural details to a resolution level of $3 \AA$ $(=0.3 \mathrm{~nm})$ [e.g., 5]. The disaccharide, sucrose, was mentioned in passing by Unwin and Henderson in their initial report on the use of glucose to prepare specimens for electron crystallography [2], but this sugar is not often applied to structural studies of protein crystals. This report evaluates the ability of sucrose to preserve protein structure during drying for TED/TEM specimen preparation.

Bovine liver catalase (Sigma) is re-crystallized into thin platelets at $\mathrm{pH} 6.3$ by progressive dilution [6]. The platelets are orthorhombic crystals with $\mathrm{a}=69 \AA, \mathrm{b}=174 \AA$, and $\mathrm{c}=206 \AA$ [7]. Suspended crystals are deposited onto a hydrophilic thin carbon support film, drained, exposed to sucrose or other experimental solution, re-drained, and then rapidly dried in air. Low-dose electron diffraction and direct imaging are conducted at $100 \mathrm{kV}$ without cryo-protection, as described [8]. All data are recorded onto SO-163 film (Kodak), which is developed in undiluted D-19 for 12 minutes.

Low-dose electron diffraction readily produces spots from catalase single crystals dried within $100 \mathrm{mM}$ sucrose. The highest angle Bragg spot recorded along the $b$-axis is at the 47 th order, corresponding to $3.7 \AA$ (Fig. 1). Use of pure water in place of sucrose gives no detectable diffraction spots. Direct imaging of catalase crystals within a sucrose matrix does not reveal any periodicities. However, when catalase crystals are dried within the potassium salt of sucrose-6-phosphate (Sigma), scattering from the elements heavier than carbon is sufficient to reveal the major $87 \AA$ periods with good contrast (Fig. 2); low-dose diffraction still shows Bragg spots out to $3.7 \AA$.

The experimental results indicate that sucrose has substantial structure-preserving activity and protects crystalline catalase from damage during drying to a high resolution level. Its covalent derivative, sucrose-monophosphate, has a comparable protective capability, and additionally acts as a light-atom negative stain that reveals lattice periodicities with good contrast in direct high-dose images. This pair of sustaining agents can provide both the diffraction amplitudes and initial phases required for reconstruction of protein structure. Sucrose thus is a good alternative to the three sugars in common use for electron crystallography. The most obvious advantage of using this disaccharide is that specimens of crystals, membranes, protein assemblies, or viruses purified on a sucrose density gradient can be prepared directly for TED/TEM without any washing or further manipulations [9]. 
[1] A. B. Richards et al., Food. Chem. Toxicol. 40 (2002) 871.

[2] P. N. T. Unwin and R. Henderson, J. Mol. Biol. 94 (1975) 425.

[3] B. K. Jap et al., J. Struct. Biol. 103 (1990) 57.

[4] W. Kühlbrandt, J. Mol. Biol. 194 (1987) 757.

[5] M. J. Ellis and H. Hebert, Micron 32 (2001) 541.

[6] N. G. Wrigley, J. Ultrastruct. Res. 24 (1968) 454.

[7] P. N. T. Unwin, J. Mol. Biol. 98 (1975) 235.

[8] W. H. Massover et al., Ultramicrosc. 90 (2001) 7.

[9] This study was supported in part by NIH grant GM57948.

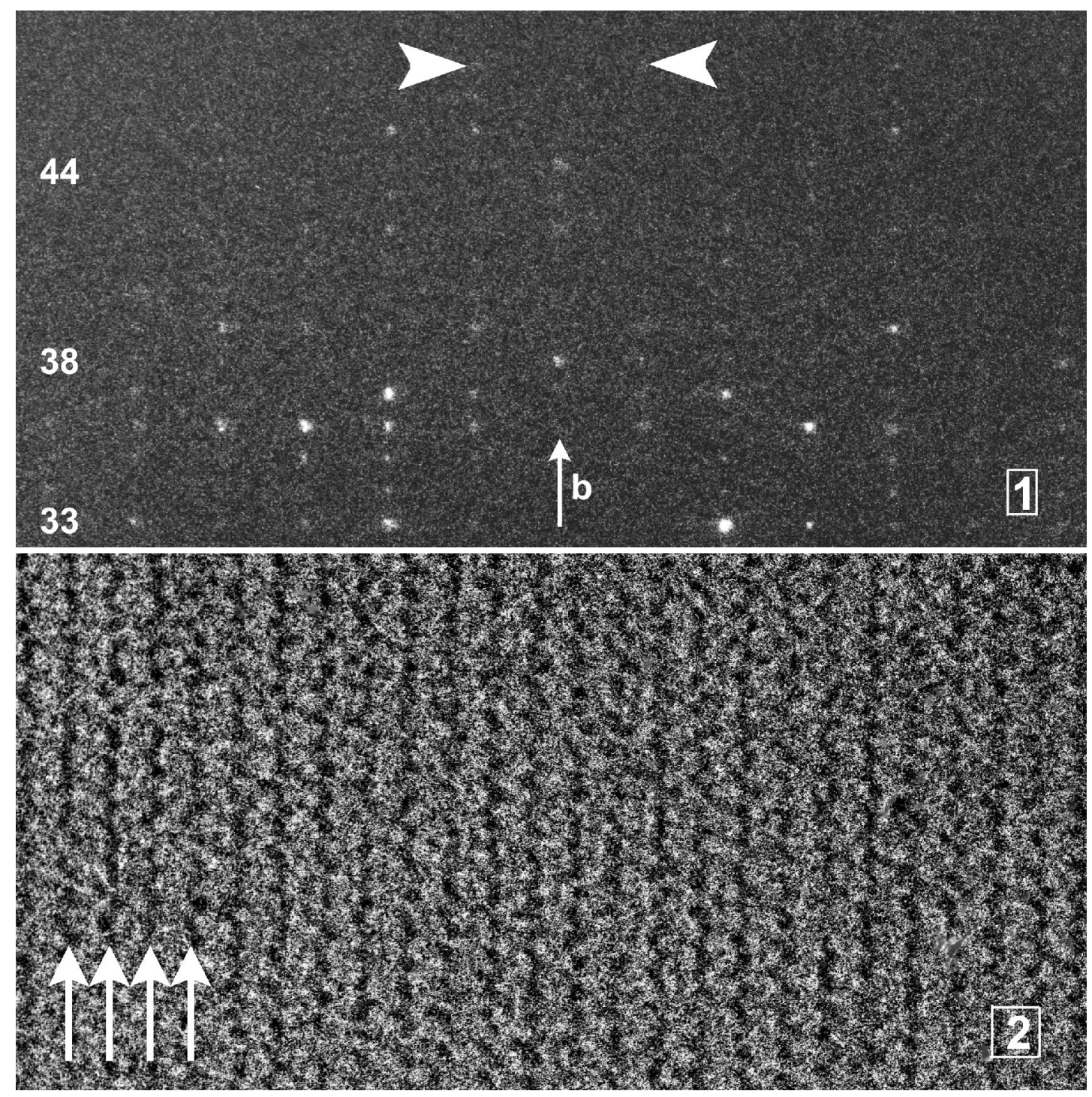

FIGURE 1: Portion of low-dose electron diffraction pattern recorded from catalase crystal dried in $100 \mathrm{mM}$ sucrose. Numbers at left indicate orders along the orthorhombic b-axis (arrow). Diffraction spots extend out to 47 orders (arrowheads at $(1,47)$ and $(-1,47)$ ). FIGURE 2: Portion of direct image of catalase crystal dried in $50 \mathrm{mM}$ sucrose-6-phosphate. $87 \AA$ major lattice periods (arrows) perpendicular to the $b$-axis are revealed with good contrast by this light-atom negative stain/sustain. 